\title{
Corporate Sustainability, Ethics and Employee Satisfaction
}

\author{
Pablo Ruiz-Palomino ${ }^{1, * \mathbb{D}}$, Rafael Morales-Sánchez ${ }^{2}$ (D) and Ricardo Martínez-Cañas ${ }^{1}$ (D) \\ 1 Business Management Department, University of Castilla-La Mancha, 16071 Cuenca, Spain; \\ ricardo.martinez@uclm.es \\ 2 Department of Business Organization and Marketing, Pablo de Olavide University, 41013 Sevilla, Spain; \\ rmorsan@upo.es \\ * Correspondence: pablo.ruiz@uclm.es; Tel.: +34-969-179-100; Fax: +34-969-179-176
}

check for updates

Citation: Ruiz-Palomino, P.; Morales-Sánchez, R.; Martínez-Cañas, R. Corporate Sustainability, Ethics and Employee Satisfaction. Sustainability 2021, 13, 11964. https:/ / doi.org/10.3390/su132111964

Received: 13 October 2021

Accepted: 27 October 2021

Published: 29 October 2021

Publisher's Note: MDPI stays neutral with regard to jurisdictional claims in published maps and institutional affiliations.
Corporate sustainability has emerged as an important area of study recently [1]. It aims to contribute to a balance among the economic, social, and environmental areas of business activities with a strong focus on how to meet stakeholders' interests [2]. Some companies do not engage in corporate sustainability as an end in itself-as integrated in corporate day-to-day functioning (does not walk its talk) — but as a means to an end-easily decoupled from corporate day-to-day functioning (i.e., public image, green and ethics washing). However, corporate sustainability must be definitively grounded on ethics to develop fair relationships with stakeholders. Thus, drawing on the 172030 UN Sustainable Development Goals, corporate sustainability should not ignore the promotion of aspects such as justice, responsible production, decent work, gender equality, good health, and well-being, among others, which lead businesses to truly consider stakeholders' interests. In line with these arguments, employees, as important stakeholders not to be ignored by corporations, must be considered ends in themselves and not as means to achieve corporate goals [3]. In doing so, corporate sustainability should promote high levels of employee satisfaction, including happiness and well-being [4]. Furthermore, inasmuch as true corporate sustainability leads employees to adopt a sense of purpose and put ethics into practice, human flourishing can emerge and thus employee satisfaction, happiness, and well-being can emerge as well.

The present Special Issue brings 7 studies by 23 authors who analyzed different research gaps concerning corporate sustainability and ethics. In particular, this Special Issue addresses three critical aspects related to the purpose and content of our Special Issue: (1) how and whether corporations really live up to their ethics and have ethics fully integrated in their day-to-day operations (Contribution 1), (2) how to reduce the occurrence of unethical pro-organizational behavior (UPB) or how to improve ethical decision making in the workplace to make organizations become sustainable in achieving positive outcomes throughout the time (Contributions 2 and 3) and (3) how corporate or management ethics/sustainable orientation can foster positive outcomes at work, including employee job satisfaction and other positive attitudes and behaviors (Contributions 4-6), or can avoid the perception of workplace stressors that may promote negative behaviors in the organization, such as UPB (Contribution 7).

With regards to the first stream of research addressed in this Special Issue-the identification of aspects that can help corporations to really live up to their ethics in their day-to-day operations and therefore "to walk the talk" in terms or corporate ethics and/or corporate sustainability - there is one paper that is very illustrative (Contribution 1). In this paper, the authors analyzed the relationship between corporate social responsibility (CSR) and earnings management (EM), or the use of accounting techniques with which to reflect a positive view of the firm's financial position. Using panel data from companies registered in the Shanghai and the Shenzhen stock exchanges, CSR engagement was found to be a negative for EM practices. The authors found that when Chinese firms increased their CSR activities, they tended to be less involved in EM activities. Importantly, they 
also revealed that when the firm was a state-owned firm, these firms were more likely to increase EM while increasing CSR activities. This could occur, according to the authors, because state-owned firms were more likely to face increased legitimacy risk. Their work reinforced previous works where firms which were engaged in CSR activities tended to improve their corporate image, and their social image improved as the stakeholder satisfaction level increased. This paper is a clear example of how higher moral standing among decision-makers can reject malpractices, such as EM, but how the type of firm (state-owned vs non-state-owned firms) and the higher likelihood of facing legitimacy risk by these firms can also influence this relationship: state-owned firms were more likely to indulge in EM activities, despite being involved in CSR activities.

Concerning the second critical stream of research addressed in this Special Issue-the identification of aspects that could help to avoid (unethical) behaviors or decision making that could harm the long-term sustainability of the organization-two papers helped to advance this realm. In the first of them (Contribution 2), Gigol endeavors to identify whether gender can be considered as an antecedent of UPB behaviors, since the literature on the subject does not show definitive results. To try to solve this research question, he carries out two studies in Poland. The first one was carried out among full-time employees of companies operating in various sectors who were extramural students $(\mathrm{N}=786)$. The second study was conducted half among employees of three large holding companies and half among working students of postgraduate studies $(\mathrm{N}=389)$. The main conclusion of this work is that, besides other factors such as Machiavellianism or an individual moral identity, gender is also a factor that differentiates employee engagement in UPB, just as it acts to explain other (ethical) behaviors in business, and the female gender (versus the male gender) is less likely to display unethical behavior for the sake of the organization. These results are consistent with the theory on UPB. In reflecting on these results, Gigol highlights the fact that the national culture may be an indirect factor influencing gender differences in engagement in UPB. As implications for management, the paper highlights women's dissimilar approach to business ethics, and their lower propensity to engage in UPB may help management boards make better and more ethical decisions in business. In conclusion, we believe these findings suggest that participation of women in the development and implementation of ethical codes may help make these codes more successful in the fulfilment of their objectives.

The other paper (Contribution 3) analyzed the role of reflective awareness in ethical decision making in business across a sample of students in Croatia. This research was conducted using undergraduates who, in the near future, were about to face ethical decision-making scenarios. The findings of this paper revealed that the decision-making process of these students was influenced by their preferred ethics. Importantly, this research concluded that organizations should not rely on the decision-making rules of employees if the aim is to improve business ethical decision making. Their findings therefore revealed that the organizations that do not have clearly defined organizational values are exposed to the risk that decisions will be made by the individuals who are not fully aware of the set of rules under which they make them, which may further result in negative outcomes. Thus, their research reinforces the idea that an improvement in reflective awareness would contribute to more sustainable ethical choices and reveals the possibility of an intervention-and-teaching design in higher education.

Finally, this Special Issue also touches on a principal theme in corporate ethics and sustainability and its effects in the workplace. Specifically, this Special Issue also includes papers that address how corporate sustainability and ethics, both reflective in the foundations and operative structure of the organization, as well as in the management and corporate structure, can foster positive outcomes at work, including employee job satisfaction. Four papers contributed to this stream of research in this Special Issue.

In one of them, the authors, using data from 352 highly skilled employees of manufacturing organizations in the Republic of Korea, demonstrated how supervisors' behavioral ethical dimensions, particularly his/her humility, could foster job satisfaction among 
employees (Contribution 5). Furthermore, their investigation revealed that abusive supervision, which includes verbal and non-verbal hostile behaviors developed by supervisors, decreased job satisfaction among the employees, which is especially critical today to successfully address the continuous challenges and complexities of the new environments which organizations must face today. Therefore, it is also critical for the sustainable functioning of any organization. Thus, in their study, these authors clarified that supervisors, by being humble, greatly help organizations to be sustainable, whereas all type of behaviors that supervisors develop to humiliate or abuse their employees is harmful to the sustainability of the organization, as the latter hampers levels of job satisfaction among employees. This study is clearly an important addition to the existing literature, as it also demonstrated that job satisfaction is a key factor in fostering employee creativity, which is critically needed to face the new complexities of the new current environment in the best possible way. In fact, and importantly, in this study, the authors demonstrated that supervisors' humility, which is in line with promoting sustainable values within the organization, was positively related to employee job satisfaction, and in turn, to employee creativity, while abusive supervision, which hampers the development of sustainable relationships within groups and organizations, was negatively related to employee job satisfaction, and in turn, was negatively related to employee creativity. Thus, job satisfaction was revealed as a significant mechanism by which corporate ethics, reflected in the specific ethical (humility) versus unethical (abusive) behavior of supervisors, is effective in driving employees to generate unique ideas, products, services or methods to overcome economic, environmental and social problems of an organization, and therefore, in promoting a strong sustainable development of the organizations in which they work.

As proof of how corporate sustainability and ethics can foster positive outcomes in the workplace, another paper (Contribution 4) demonstrated that worker-owned cooperative organizations can foster employee wellbeing in the form of employee job satisfaction. In their study, these authors analyzed how the percentage of employees who are owners of the cooperative in which they are working was a critical factor in encouraging a helping climate that, in turn, can boost workplace participation to ultimately foster workplace satisfaction. This paper thus revealed that in employee co-operatives-labor owned and managed entities-where resources are available and usually utilized to fulfill common interests among employee owners, positive outcomes are more likely than in those organizations (cooperatives) where having employee owners is not the norm. The analyses conducted in this study on a sample of 5907 individuals working in 99 different hypermarkets of a large Spanish cooperative retail chain revealed that positive outcomes (i.e., a helping climate, workplace participation, job satisfaction) typically associated with cooperative values decreased when the percentage of waged and temporary employees was high. The results instead revealed that a higher share of co-owners in these hypermarkets positively related to workplace satisfaction via sequentially generating stronger helping climates (e.g., peer support and help perceived in the workplace) and stronger workplace participation (e.g., participation in key decisions related to plans and strategies that affect the workplace). This study is therefore a clear example of how some deviations (i.e., hiring workers who are not co-owners) from the foundational sustainability principles of worker-owned cooperative organizations could negatively affect the organization functioning in such a way that lower levels of job satisfaction could emerge. Thus, the paper is an example of how not living the sustainability principles that, from a foundational perspective, an organization is expected to live and that formally declares to live-in other words, not walking the talk-could be seriously detrimental to several aspects that are considered critical to the sustainable performance of any organization, including workplace satisfaction.

Continuing with the analysis of business practices that promote business sustainability specified in the well-being of employees, the paper of Peeters, Van De Voorde and Paauwe (Contribution 6) attempts to delve into the different relationships that exist between energy levels and employee performance, and investigates the antecedents of these relationships. This research is important and novel, because managing a company in a sustainable way 
implies obtaining good economic (e.g., profitability) and social (e.g., employee happiness) performance outcomes at the same time. Traditionally, it has been considered that the variables of employee well-being (satisfaction, happiness, ... ) were antecedents of high performance (through commitment to the organization or other mediating mechanisms). However, as Peeters, Van De Voorde and Paauwe remind us, these relationships are not the only ones that can exist in organizations, since previous studies have shown the existence of five dyads: 1 . Low well-being/low performance; 2 . Low well-being/medium performance; 3. High well-being/medium performance; 4 . High well-being/high performance; and 5. High well-being/top performance. This research not only tries to show the relationships that exist between vitality and performance through a study that uses multinomial regression for a sample of 5729 employees from the Dutch division of a large bank, it also explores the antecedents of these relationships to try to shed light on the mechanisms that can facilitate a win-win management strategy that leads to the enhancement of both financial performance and employee happiness outcomes. As each profile appears to be predicted by distinct job demands and resources, this study sheds light on the difficult task of creating jobs that are beneficial to both organizations and their employees. This means that managers - preferably in close cooperation with their HR business manager-should consider these complex employee well-being/performance patterns when deciding upon an intervention.

Finally, one last paper (Contribution 7) was also very illustrative of the factors that drive the development of unethical pro-organizational behavior (UPB) - a type of behavior that, despite pursuing the fostering of the effective functioning of the organization, violates laws and social and ethical values (Contribution 7) [5] —in the workplace. The fact that this type of behavior can be developed in the workplace is seriously detrimental to the reputation of an organization, and is likely to hinder the proper and adequate development of that organization in the long term, thus becoming a barrier to the sustainability of the organization. In their article, using data from 375 employees of 6 enterprises in Nanjing and Shanghai, China, these authors demonstrated that certain stressors that are usually derived from unethical aspects originated from the top of the organization (i.e., job insecurity, ethical and performance pressure) could be main drivers of this type of unethical behavior. These authors thus demonstrated that if poor sustainable values are printed in the climate and functioning of the organization and stress is generated among the employees, even if it is in a very unconscious way, the development of unethical behavior by employees is highly probable, and therefore the sustainable operation of the organization may be in serious danger. New to the literature, the authors demonstrated the differentiated effects of distinct stressors on UPB and the distinct mechanisms that underlie these effects. In their investigation, they demonstrated that all stressors that are considered as an injury or potential loss to individuals (i.e., hindrance stressors such as role conflict or threats to individuals' existing resources) encouraged employees to develop higher levels of UPB through elevating their levels of anger and anxiety, respectively. However, all stressors that could be seen as an opportunity to grow or to learn (i.e., challenge stressors such as workload, job complexity or time urgency) were observed to limit the development of UPB by mainly activating feelings of alertness, concentration and determination among the employees. It is true that anxiety was also higher when any of these stressors were perceived, and that therefore, these two types of stressors favoured the development of UPB via the higher level of anxiety provoked among the employees. However, the results in this paper clarified that while management should design strategies that can help reduce the appearance of all type of stressors in organizations, they should pay significant attention to reducing the appearance of hindrance stressors, as these stressors were found to be the most impactful in provoking UPB among the employees. In fact, their results demonstrated that the total effect of challenge stressors on UPB was practically non-existent. The results of this paper are therefore very enlightening regarding how to avoid a series of behaviors (i.e., UPBs) that can be very harmful for the sustainable development of business organizations. 
This editorial introduction was aimed at expressing the importance of the topic selected and at presenting the content of this special. As explained, the participating authors addressed different aspects that help advance the existing literature on the links existing between corporate sustainability, ethics and employee satisfaction. These include (i) the identification of aspects (engagement in CSR activities) that can help corporations not to fall into unethical practices, (ii) the identification of aspects that can help to avoid (unethical) decision making (female gender, clearly defined values, and hiring for reflective awareness) and (iii) inquiries on how corporate sustainability and ethics foster positive satisfactionand well-being-related outcomes. The findings of this Special Issue lead us to think that there are factors that can impede organizations from living up to their ethics and that "walking the talk" is critical to obtain benefits that can lead firms to be sustainable.

\section{List of Contributions:}

1. Chen, X.; Sial, M.S.; Tran, D.K.; Alhaddad, W.; Hwang, J.; Thu, P.A. Are socially responsible companies really ethical? The moderating role of state-owned enterprises: Evidence from China.

2. Gigol, T. Gender differences in engagement in unethical pro-organizational behavior-two studies in Poland.

3. Božac, M.G.; Kostelić, K.; Paulišić, M.; Smith, C.G. Business ethics decision-making: Examining partial reflective awareness.

4. De Reuver, R.; Kroon, B.; Olabarria, D.M.; Iñurritegui, U.E. Employee satisfaction in laborowned and managed workplaces: Helping climate and participation spillover to non-owners.

5. Miao, S.; Fayzullaev, A.K.; Dedahanov, A.T. Management characteristics as determinants of employee creativity: The mediating role of employee job satisfaction.

6. Peeters, T.; Van De Voorde, K.; Paauwe, J. Exploring the nature and antecedents of employee energetic well-being at work and job performance profiles.

7. $\mathrm{Xu}, \mathrm{L} . ;$ behavior: Mediating role of emotions.

Funding: This research received no external funding.

Conflicts of Interest: The authors declare no conflict of interest.

\section{References}

1. Nikolaou, I.E.; Tsalis, T.A.; Evangelinos, K.I. A framework to measure corporate sustainability performance: A strong sustainability-based view of firm. Sustain. Prod. Consum. 2019, 18, 1-18. [CrossRef]

2. Schrippe, P.; Ribeiro, J.L.D. Preponderant criteria for the definition of corporate sustainability based on Brazilian sustainable companies. J. Clean. Prod. 2019, 209, 10-19. [CrossRef]

3. Melé, D. Business Ethics in Action: Seeking Human Excellence in Organizations; Palgrave Macmillan: London, UK, 2009; ISBN 9780230573109.

4. Chamberlin, A.; Zhao, D. The Key to Happy Customers? Happy Employees. Harv. Bus. Rev. 2019. Available online: https:/ /hbr.org/2019/08/the-key-to-happy-customers-happy-employees (accessed on 15 July 2021).

5. Umphress, E.E.; Bingham, J.B. When employees do bad things for good reasons: Examining unethical pro-organizational behaviors. Organ. Sci. 2011, 22, 621-640. [CrossRef] 